\title{
A rigorous variant of the shear strength reduction method and its geotechicalapplications
}

\author{
Stanislav Sysala*, Eva Hrubešová ${ }^{\dagger, *}$, ZdeněkMichalec* and Franz Tschuchnigg \\ * Institute of Geonics of the Czech Academy of Sciences \\ Studentska 1768, 708 00Ostrava, Czech Republic \\ e-mails: stanislav.sysala@ugn.cas.cz,zd.michalec@volny.cz,web page: http://www.ugn.cas.cz \\ ${ }^{\dagger}$ VSB-Technical University Ostrava, Faculty of Civil Engineering \\ 17.listopadu 15, 70800 Ostrava, Czech Republic \\ Email: eva.hrubesova@vsb.cz,web page: http://www.fast.vsb.cz \\ Graz University of Technology \\ Institute of Soil Mechanics, Foundation Engineering and Computational Geotechnics \\ Rechbauerstraße 12,8010 Graz, Austria \\ email: franz.tschuchnigg@tugraz.at, web page: graz.pure.elsevier.com/en/
}

\begin{abstract}
This contributionis focused on a rigorous variant of the shear strength reduction method (SSR) and the corresponding determination of safety factors. It summarizes results presented in [1] and is inspired by [2]. The SSR-based safety factor is proposed to define as a solution of an optimization problem that is independent of the plastic flow rule and the space discretization. In case of nonassociative plasticity, a modified Davis approach is used. The optimization problem is analyzed and the corresponding duality between the static and kinematic principles is derived. For numerical solution, a regularization method is introduced and a relation between the original and regularized problems is derived. The regularization method is combined with the finite element method, mesh adaptivity and a damped Newton method. In-house codes in Matlabare used for implementation of this solution concept. Two slope stability problems are considered, one of which follows from analysis of a real slope. SoftwaresPlaxis and ComsolMultiphysicsare used for comparison of the results. We also discuss uncertainty treatment and an influence of the porous flow.
\end{abstract}

\section{REFERENCES}

[1] Sysala, S., Hrubesova, E., Michalec, Z. andTschuchnigg, F. Optimization and variational principles for the shear strength reduction method. Submitted(2021), https://arxiv.org/pdf/2101.01005.pdf.

[2] Tschuchnigg, F., Schweiger, H.F.and Sloan, S.W.. Slope stability analysis by means of finite element limit analysis and finite element strength reduction techniques. Part I: Numerical studies considering non-associated plasticity. Computers and Geotechnics, 70 (2015)169-177. 